\title{
ON CERTAIN MARTINGALE INEQUALITIES FOR MAXIMAL FUNCTIONS AND MEAN OSCILLATIONS
}

\author{
MASATO KIKUCHI and YASUHIRO KINOSHITA*
}

\begin{abstract}
Let $X$ be a Banach function space over a nonatomic probability space. For a uniformly integrable martingale $f=\left(f_{n}\right)$ with respect to a filtration $\mathscr{F}=\left(\mathscr{F}_{n}\right)$, let $M f=\sup _{n}\left|f_{n}\right|$ and $\theta_{\mathscr{F}} f=$ $\sup _{n} E\left[\left|f_{\infty}-f_{n-1}\right| \mid \mathscr{F}_{n}\right]$. We give a necessary and sufficient condition on $X$ for the inequality $\left\|\theta_{\mathscr{F}} f\right\|_{X} \leq C\|M f\|_{X}$ to hold.
\end{abstract}

\section{Introduction}

Let $(\Omega, \Sigma, P)$ be a nonatomic probability space and let $\mathrm{F}$ be the collection of all filtrations, where by a filtration we mean a sequence $\mathscr{F}=\left(\mathscr{F}_{n}\right)_{n \in Z_{+}}$of sub- $\sigma$-algebras of $\Sigma$ such that $\mathscr{F}_{n} \subset \mathscr{F}_{n+1}$ for all $n \in \mathrm{Z}_{+}$. Given $\mathscr{F}=\left(\mathscr{F}_{n}\right) \in$ F, let $\mathscr{M}_{u}(\mathscr{F})$ denote the collection of all (real-valued) uniformly integrable martingales with respect to $\mathscr{F}$ and $P$. For each $f=\left(f_{n}\right) \in \mathscr{M}_{u}(\mathscr{F})$, let $f_{\infty}$ denote the almost sure limit of $f$, and let $f_{-1} \equiv 0$. Following [5], we define

$$
\theta_{\mathscr{F}} f=\sup _{0 \leq n<\infty} E\left[\left|f_{\infty}-f_{n-1}\right| \mid \mathscr{F}_{n}\right] .
$$

(Some authors use the notation $f^{\sharp}$ for our $\theta_{\mathscr{F}} f$.) Various inequalities for $\theta_{\mathscr{F}} f$ have been established. For example, Lépingle [6] proved that if $\Phi:[0, \infty) \rightarrow$ $[0, \infty)$ is a continuous increasing function such that $\Phi(0)=0$ and $\sup _{t>0}(\Phi(a t) / \Phi(t))<\infty$ for some $a>1$, then there is a constant $c$ such that for every $f=\left(f_{n}\right) \in \mathscr{M}_{u}(\mathscr{F})$ with $f_{0}=0$ a.s.,

$$
E[\Phi(M f)] \leq c E\left[\Phi\left(\theta_{\mathscr{F}} f\right)\right],
$$

where $M f$ denotes the maximal function of $f$, i.e.,

$$
M f=\sup _{0 \leq n<\infty}\left|f_{n}\right|
$$

* The first author was supported by JSPS Grant-in-Aid for Scientific Research (C) 20540160.

Received 15 April 2010. 
Furthermore, Long [7] established some extensions of Lépingle's result by using rearrangement inequalities. For some other results, see [4], [5], [8], and so on.

Let $X$ be a Banach function space over $\Omega$ (see Definition 1). Recall that $X$ is called a rearrangement-invariant function space (ri. space) if the norm of each $x \in X$ is determined by its distribution (see Definition 2). The main theorem of [5] says that the following conditions on $X$ are equivalent:

(i) There exist constants $c=c(X)$ and $C=C(X)$ such that if $\mathscr{F} \in \mathrm{F}$ and $f \in \mathscr{M}_{u}(\mathscr{F})$, then

$$
c\left\|f_{\infty}\right\|_{X} \leq\left\|\theta_{\mathscr{F}} f\right\|_{X} \leq C\left\|f_{\infty}\right\|_{X} .
$$

(ii) $X$ can be equivalently renormed so as to be an r.i. space such that $0<$ $\alpha_{X} \leq \beta_{X}<1$.

Here $\alpha_{X}$ and $\beta_{X}$ denote the lower and upper Boyd indices of $X$, respectively.

Moreover, one can deduce from [5, Proposition 3] that if $X$ is an r.i. space and $\alpha_{X}>0$, then there is a constant $C=C(X)$ such that for every $f \in \mathscr{M}_{u}(\mathscr{F})$,

$$
\|M f\|_{X} \leq C\left\|\theta_{\mathscr{F}} f\right\|_{X} .
$$

Thus if $X$ can be equivalently renormed so as to be an ri. space such that $0<$ $\alpha_{X} \leq \beta_{X}<1$, then (1) holds with $f_{\infty}$ replaced by $M f$. It is therefore natural to ask whether the converse is true, i.e., to ask whether $X$ can be equivalently renormed so as to be an r.i. space such that $0<\alpha_{X} \leq \beta_{X}<1$ when (1) holds with $f_{\infty}$ replaced by $M f$. The affirmative answer (see Corollary 2) will follow from the main results of the present paper.

\section{Preliminaries}

Throughout the paper, we assume that the probability space $(\Omega, \Sigma, P)$ is nonatomic, i.e., there is no $P$-atom in $\Omega$.

We denote by $I$ the interval $(0,1]$ and consider $I$ as a probability space equipped with Lebesgue measure $d s$.

Given two Banach spaces $X_{1}$ and $X_{2}$, we write $X_{1} \hookrightarrow X_{2}$ to mean that $X_{1}$ is continuously embedded in $X_{2}$.

DEFINITION 1. A Banach function space $X$ over a probability space is a real Banach space of (equivalence classes of) random variables (in other words, measurable functions) which satisfies the following conditions:

(B1) $L_{\infty} \hookrightarrow X \hookrightarrow L_{1}$.

(B2) If $|y| \leq|x|$ a.s. and $x \in X$, then $y \in X$ and $\|y\|_{X} \leq\|x\|_{X}$. 
(B3) If $0 \leq x_{n} \uparrow x$ a.s., $x_{n} \in X$ for all $n$, and $\sup _{n}\left\|x_{n}\right\|_{X}<\infty$, then $x \in X$ and $\|x\|_{X}=\sup _{n}\left\|x_{n}\right\|_{X}$.

We adopt the convention that $\|x\|_{X}=\infty$ for every random variable $x$ which does not belong to $X$.

Given two random variables $x$ and $y$, we write $x \simeq_{d} y$ to mean that they have the same distribution.

Definition 2. A rearrangement-invariant function space (ri. space) $X$ over a probability space is a Banach function space which satisfies the following condition:

(RI) If $x \simeq_{d} y$ and $x \in X$, then $y \in X$ and $\|y\|_{X}=\|x\|_{X}$.

For example, Lebesgue spaces, Orlicz spaces, and Lorentz spaces are r.i. spaces. On the other hand, Lebesgue spaces with suitable weights are Banach function spaces which are not r.i. spaces.

Let $x$ be a random variable on $\Omega$. The nonincreasing rearrangement of $x$, which is denoted by $x^{*}$, is a unique nonincreasing right-continuous function on $I=(0,1]$ whose distribution (with respect to Lebesgue measure) is the same as that of $|x|$. Note that $x^{*}$ can be written as

$$
x^{*}(t)=\inf \{\lambda>0 \mid P(|x|>\lambda) \leq t\}, \quad t \in I,
$$

with the convention that inf $\emptyset=\infty$. The nonincreasing rearrangement $\phi^{*}$ of a measurable function $\phi$ on $I$ is defined in the same way.

Let $X$ be an r.i. space over $\Omega$. Then there is a unique r.i. space $\widehat{X}$ over $I$ such that:

- $x \in X$ if and only if $x^{*} \in \widehat{X}$.

- $\|x\|_{X}=\left\|x^{*}\right\|_{\widehat{X}}$ for every $x \in X$.

We call $\widehat{X}$ the Luxemburg representation of $X$. For example, the Luxemburg representation of $L_{p}(\Omega)$ is $L_{p}(I)$. See [1, pp. 62-64] or [9, (12.2), p. 121] for a proof.

Let $L_{0}(I)$ denote the space of all measurable functions on $I$, and let $Z_{1}$ and $Z_{2}$ be two Banach function spaces over $I$. We denote by $B\left(Z_{1}, Z_{2}\right)$ the collection of all linear operators $T$ such that:

- The domain of $T$ contains $Z_{1}$ and the range of $T$ is contained in $L_{0}(I)$.

- The restriction of $T$ to $Z_{1}$ is a bounded operator from $Z_{1}$ into $Z_{2}$.

For each $T \in B\left(Z_{1}, Z_{2}\right)$, we denote by $\|T\|_{B\left(Z_{1}, Z_{2}\right)}$ the operator norm of the restriction of $T$ to $Z_{1}$. In the case where $Z=Z_{1}=Z_{2}$, we write $B(Z)$ for $B(Z, Z)$. 
We now recall the definition of Boyd indices. For each $s>0$, define an operator $D_{s}: L_{0}(I) \rightarrow L_{0}(I)$ by

$$
\left(D_{s} \phi\right)(t)= \begin{cases}\phi(s t) & \text { if } s t \in I, \\ 0 & \text { otherwise. }\end{cases}
$$

If $Z$ is an r.i. space over $I$, then $D_{s} \in B(Z)$ for every $s>0$, and the lower and upper Boyd indices of $Z$ are defined by

$$
\alpha_{Z}=\sup _{0<s<1} \frac{\log \left\|D_{1 / s}\right\|_{B(Z)}}{\log s} \quad \text { and } \quad \beta_{Z}=\inf _{1<s<\infty} \frac{\log \left\|D_{1 / s}\right\|_{B(Z)}}{\log s},
$$

respectively. If $X$ is an r.i. space over $\Omega$, then the Boyd indices of $X$ are defined by $\alpha_{X}=\alpha_{\widehat{X}}$ and $\beta_{X}=\beta_{\widehat{X}}$. For example, $\alpha_{L_{p}}=\beta_{L_{p}}=1 / p$ for every $p \in[1, \infty]$. It is well known that $0 \leq \alpha_{X} \leq \beta_{X} \leq 1$ for every r.i. space $X$. See e.g. [1, pp. 146-150] for details.

In order to prove our results, we will need to discuss the boundedness of the averaging operator $\mathscr{P}$ and its (formal) adjoint $\mathscr{Q}$. Recall that $\mathscr{P}$ is defined for $\phi \in L_{0}(I)$ by

$$
(\mathscr{P} \phi)(t)=\frac{1}{t} \int_{0}^{t} \phi(s) d s, \quad t \in I,
$$

and $\mathscr{Q}$ is defined for $\phi \in L_{0}(I)$ by

$$
(\mathscr{Q} \phi)(t)=\int_{t}^{1} \frac{\phi(s)}{s} d s, \quad t \in I,
$$

provided that the respective integrals are defined and finite for all $t \in I$. Suppose that $X$ is an r.i. space over $\Omega$. Then $\beta_{X}<1$ if and only if $\mathscr{P} \in B(\widehat{X})$, and $\alpha_{X}>0$ if and only if $\mathscr{Q} \in B(\widehat{X})$ (see [1, Theorem 5.15, p. 150]; cf. [10]).

\section{Statement of results}

The main theorem of this paper is

THEOREM 1. Let $X$ be a Banach function space over $\Omega$. Then the following are equivalent:

(i) There exists a constant $C=C(X)$ such that if $\mathscr{F} \in \mathrm{F}$ and $f \in \mathscr{M}_{u}(\mathscr{F})$, then

$$
\left\|\theta_{\mathscr{F}} f\right\|_{X} \leq C\|M f\|_{X} .
$$

(ii) There exists a constant $C=C(X)$ such that if $\mathscr{F} \in \mathrm{F}$ and $f \in \mathscr{M}_{u}(\mathscr{F})$, then

$$
\left\|\theta_{\mathscr{F}} f\right\|_{X} \leq C \sup _{0 \leq n<\infty}\left\|f_{n}\right\|_{X} .
$$


(iii) There exists a constant $C=C(X)$ such that if $\mathscr{F} \in \mathrm{F}$ and $f \in \mathscr{M}_{u}(\mathscr{F})$, then

$$
\|\theta \mathscr{F} f\|_{X} \leq C\left\|f_{\infty}\right\|_{X} .
$$

(iv) $X$ can be equivalently renormed so as to be an ri. space such that $\beta_{X}<1$.

The following result extends the main theorem of [5].

Corollary 2. Let $X$ be a Banach function space over $\Omega$. Then the following are equivalent:

(i) There exist constants $c=c(X)$ and $C=C(X)$ such that if $\mathscr{F} \in \mathrm{F}$ and $f \in \mathscr{M}_{u}(\mathscr{F})$, then

$$
c\|M f\|_{X} \leq\|\theta \mathscr{F} f\|_{X} \leq C\|M f\|_{X} .
$$

(ii) There exist constants $c=c(X)$ and $C=C(X)$ such that if $\mathscr{F} \in \mathrm{F}$ and $f \in \mathscr{M}_{u}(\mathscr{F})$, then

$$
c \sup _{0 \leq n<\infty}\left\|f_{n}\right\|_{X} \leq\left\|\theta_{\mathscr{F}} f\right\|_{X} \leq C \sup _{0 \leq n<\infty}\left\|f_{n}\right\|_{X} .
$$

(iii) There exist constants $c=c(X)$ and $C=C(X)$ such that if $\mathscr{F} \in \mathrm{F}$ and $f \in \mathscr{M}_{u}(\mathscr{F})$, then

$$
c\left\|f_{\infty}\right\|_{X} \leq\left\|\theta_{\mathscr{F}} f\right\|_{X} \leq C\left\|f_{\infty}\right\|_{X} .
$$

(iv) $X$ can be equivalently renormed so as to be an ri. space such that $0<\alpha_{X} \leq \beta_{X}<1$.

In the proof of Theorem 1, we will use the following

Proposition 3. Let $X$ and $Y$ be two ri. spaces over $\Omega$. Then the following are equivalent:

(i) There exists a constant $C=C(X, Y)$ such that if $\mathscr{F} \in \mathrm{F}$ and $f \in$ $\mathcal{M}_{u}(\mathscr{F})$, then

$$
\left\|\theta_{\mathscr{F}} f\right\|_{X} \leq C\|M f\|_{Y} .
$$

(ii) There exists a constant $C=C(X, Y)$ such that if $\mathscr{F} \in \mathrm{F}$ and $f \in$ $\mathcal{M}_{u}(\mathscr{F})$, then

$$
\left\|\theta_{\mathscr{F}} f\right\|_{X} \leq C \sup _{0 \leq n<\infty}\left\|f_{n}\right\|_{Y} .
$$

(iii) There exists a constant $C=C(X, Y)$ such that if $\mathscr{F} \in \mathrm{F}$ and $f \in$ $\mathcal{M}_{u}(\mathscr{F})$, then

$$
\left\|\theta_{\mathscr{F}} f\right\|_{X} \leq C\left\|f_{\infty}\right\|_{Y} .
$$

(iv) $\mathscr{P} \in B(\widehat{Y}, \widehat{X})$. 


\section{Proofs}

Let us first prove Proposition 3. We will need three lemmas.

Lemma 1. Let $\phi \in L_{1}(I)$ be a nonnegative function. Then there exist nonnegative random variables $z_{1}$ and $z_{2}$, and families of sets $\left\{A_{1}(t) \in \Sigma \mid 0<\right.$ $t \leq 1 / 2\}$ and $\left\{A_{2}(t) \in \Sigma \mid 0<t \leq 1 / 2\right\}$ such that:

(i) $\left\{z_{1}>0\right\} \subset A_{1}(1 / 2)$ and $\left\{z_{2}>0\right\} \subset A_{2}(1 / 2)$.

(ii) $A_{1}(1 / 2) \cap A_{2}(1 / 2)=\emptyset$.

(iii) $A_{1}(s) \subset A_{1}(t)$ and $A_{2}(s) \subset A_{2}(t)$ whenever $0<s<t \leq 1 / 2$.

(iv) $P\left(A_{1}(t)\right)=P\left(A_{2}(t)\right)=t$ whenever $0<t \leq 1 / 2$.

(v) $E\left[z_{1} 1_{A_{1}(t)}\right]=E\left[z_{2} 1_{A_{2}(t)}\right]=t(\mathscr{P} \phi)(2 t)$ whenever $0<t \leq 1 / 2$.

(vi) $\left(z_{1}-z_{2}\right)^{*}=\left(z_{1}+z_{2}\right)^{*}=\phi^{*}$ on $I$.

Here $1_{A}$ denotes the indicator function of a set $A$.

Proof. Since $\Omega$ is nonatomic, we can use [2, (5.6), p. 44] to find nonnegative random variables $\xi_{1}$ and $\xi_{2}$ such that:

- $\left\{\xi_{1}>0\right\} \cap\left\{\xi_{2}>0\right\}=\emptyset$.

- $\xi_{1}^{*}(t)=\xi_{2}^{*}(t)=\max \{1-2 t, 0\}$ for every $t \in I$.

Define $z_{1}$ and $z_{2}$ by setting

$$
z_{1}=\phi\left(1-\xi_{1}\right) 1_{\left\{\xi_{1}>0\right\}} \quad \text { and } \quad z_{2}=\phi\left(1-\xi_{2}\right) 1_{\left\{\xi_{2}>0\right\}},
$$

and define $\left\{A_{1}(t) \in \Sigma \mid 0<t \leq 1 / 2\right\}$ and $\left\{A_{2}(t) \in \Sigma \mid 0<t \leq 1 / 2\right\}$ by setting

$$
A_{1}(t)=\left\{\xi_{1}>1-2 t\right\} \quad \text { and } \quad A_{2}(t)=\left\{\xi_{2}>1-2 t\right\} .
$$

Then it is straightforward to check that the required conditions are satisfied.

If $Z$ is a Banach function space over $I$, we let $\mathscr{D}(Z)$ denote the collection of all functions in $Z$ which are nonnegative, nonincreasing, and right-continuous, and we let $\mathscr{D}^{\prime}(Z)=\mathscr{D}(Z) \backslash L_{\infty}(I)$.

Lemma 2. Let $Z_{1}$ and $Z_{2}$ be two ri. spaces over I. Suppose $Z_{1} \neq L_{\infty}(I)$ and suppose there is a constant $c=c\left(Z_{1}, Z_{2}\right)$ such that for every $\phi \in \mathscr{D}^{\prime}\left(Z_{1}\right)$,

$$
\|\mathscr{P} \phi\|_{Z_{2}} \leq c\|\phi\|_{Z_{1}} .
$$

Then $\mathscr{P} \in B\left(Z_{1}, Z_{2}\right)$. 
Remark. Note that if $Z_{1}=L_{\infty}(I)$, then $\mathscr{P} \in B\left(Z_{1}, Z_{2}\right)$ for every Banach function space $Z_{2}$ over $I$. This follows immediately from the fact that $\mathscr{P} \in$ $B\left(L_{\infty}(I)\right)$ and $L_{\infty}(I) \hookrightarrow Z_{2}$.

Proof of Lemma 2. By [5, Lemma 3], it suffices to show that (2) holds for every $\phi \in \mathscr{D}\left(Z_{1}\right) \cap L_{\infty}(I)=\mathscr{D}\left(L_{\infty}(I)\right)$. Let $\psi \in \mathscr{D}^{\prime}\left(Z_{1}\right)$ and $\varepsilon>0$. Applying (2) to $\phi+\varepsilon \psi$ and letting $\varepsilon \rightarrow 0+$, we see that (2) holds for this $\phi$, as required.

Lemma 3. If $\mathscr{F} \in \mathrm{F}$ and $f \in \mathscr{M}_{u}(\mathscr{F})$, then $(M f)^{*} \leq \mathscr{P} f_{\infty}^{*}$ on I.

Proof. This is an almost direct corollary of Doob's maximal inequality ([8, p. 34]). See the proof of [3, Proposition 3] or of [4, Theorem 4.1] for details.

We are now ready to prove Proposition 3.

Proof of Proposition 3. (iv) $\Rightarrow$ (iii) Suppose $\mathscr{P} \in B(\widehat{Y}, \widehat{X})$. Let $\mathscr{F}=$ $\left(\mathscr{F}_{n}\right) \in \mathrm{F}$, let $f=\left(f_{n}\right) \in \mathscr{M}_{u}(\mathscr{F})$, and let $\tilde{f}=\left(\widetilde{f}_{n}\right)$ denote the martingale defined by $\widetilde{f}_{n}=E\left[\left|f_{\infty}\right| \mid \mathscr{F}_{n}\right]$. It is then clear that $\theta_{\mathscr{F}} f \leq 2 M \widetilde{f}$. Since $\widetilde{f}_{\infty}=$ $\left|f_{\infty}\right|$ a.s., it follows from Lemma 3 that $\left(\theta_{\mathscr{F}} f\right)^{*} \leq 2 \mathscr{P} \tilde{f}_{\infty}^{*}=2 \mathscr{P} f_{\infty}^{*}$ on $I$. Hence

$$
\begin{aligned}
\left\|\theta_{\mathscr{F}} f\right\|_{X}=\left\|\left(\theta_{\mathscr{F}} f\right)^{*}\right\|_{\widehat{X}} & \leq 2\left\|\mathscr{P} f_{\infty}^{*}\right\|_{\widehat{X}} \\
& \leq 2\|\mathscr{P}\|_{B(\widehat{Y}, \widehat{X})}\left\|f_{\infty}^{*}\right\|_{\widehat{Y}}=2\|\mathscr{P}\|_{B(\widehat{Y}, \widehat{X})}\left\|f_{\infty}\right\|_{Y} .
\end{aligned}
$$

This shows that (iv) implies (iii).

(iii) $\Rightarrow$ (ii) If $f=\left(f_{n}\right)$ is a uniformly integrable martingale, then by (B3) with $X$ replaced by $Y$,

$$
\left\|f_{\infty}\right\|_{Y}=\left\|\lim _{n \rightarrow \infty}\left|f_{n}\right|\right\|_{Y} \leq \varliminf_{n \rightarrow \infty}\left\|f_{n}\right\|_{Y} \leq \sup _{0 \leq n<\infty}\left\|f_{n}\right\|_{Y} .
$$

From this fact it is clear that (iii) implies (ii).

(ii) $\Rightarrow$ (i) Obvious from (B2) with $X$ replaced by $Y$.

(i) $\Rightarrow$ (iv) Assume that (i) holds. As remarked above, if $\widehat{Y}=L_{\infty}(I)$, then $\mathscr{P} \in B(\widehat{Y}, \widehat{X})$. Thus we may assume $\widehat{Y} \neq L_{\infty}(I)$. By Lemma 2 , it suffices to show that there is a constant $c=c(X, Y)$ such that $\|\mathscr{P} \phi\|_{\widehat{X}} \leq c\|\phi\|_{\widehat{Y}}$ for every $\phi \in \mathscr{D}^{\prime}(\widehat{Y})$.

Suppose $\phi \in \mathscr{D}^{\prime}(\widehat{Y})$. Then $\mathscr{P} \phi$ is strictly decreasing and continuous. Moreover $(\mathscr{P} \phi)(t) \rightarrow \infty$ as $t \rightarrow 0+$. Hence there is a strictly decreasing sequence $\left\{t_{n}\right\}_{n \in Z_{+}}$of numbers in $(0,1 / 2]$ such that $t_{0}=1 / 2$ and

$$
(\mathscr{P} \phi)\left(2 t_{n+1}\right)=2(\mathscr{P} \phi)\left(2 t_{n}\right) \quad \text { for every } n \in Z_{+} .
$$

It is clear that $t_{n} \rightarrow 0$ as $n \rightarrow \infty$. Let $z_{1}, z_{2},\left\{A_{1}(t) \in \Sigma \mid 0<t \leq 1 / 2\right\}$, and $\left\{A_{2}(t) \in \Sigma \mid 0<t \leq 1 / 2\right\}$ be as in Lemma 1 , and let $z=z_{1}-z_{2}$. 
Then $z^{*}=\phi$ on $I$. Write $\Lambda_{n}=A_{1}\left(t_{n}\right) \cup A_{2}\left(t_{n}\right)$ for each $n \in \mathrm{Z}_{+}$. Define $\mathscr{F}=\left(\mathscr{F}_{n}\right) \in \mathrm{F}$ by

$$
\mathscr{F}_{n}=\sigma\left\{\Lambda \backslash \Lambda_{n} \mid \Lambda \in \Sigma\right\}, \quad n \in \mathrm{Z}_{+},
$$

and define $f=\left(f_{n}\right) \in \mathscr{M}_{u}(\mathscr{F})$ by

$$
f_{n}=E\left[z \mid \mathscr{F}_{n}\right]=z 1_{\Omega \backslash \Lambda_{n}}, \quad n \in \mathrm{Z}_{+} .
$$

If $n \geq 1$, then

$$
\begin{aligned}
E\left[\mid f_{\infty}\right. & \left.-f_{n-1}|| \mathscr{F}_{n}\right] \\
& =\frac{1_{\Lambda_{n}}}{P\left(\Lambda_{n}\right)} E\left[|z| 1_{\Lambda_{n}}\right]+|z| 1_{\Lambda_{n-1} \backslash \Lambda_{n}} \\
& \geq \frac{1_{\Lambda_{n}}}{2 t_{n}}\left(E\left[z_{1} 1_{A_{1}\left(t_{n}\right)}\right]+E\left[z_{2} 1_{A_{2}\left(t_{n}\right)}\right]\right)=(\mathscr{P} \phi)\left(2 t_{n}\right) 1_{\Lambda_{n}} \quad \text { a.s., }
\end{aligned}
$$

and if $n=0$, then

$$
E\left[\left|f_{\infty}-f_{n-1}\right| \mid \mathscr{F}_{n}\right]=E\left[|z| \mid \mathscr{F}_{0}\right]=\|z\|_{L_{1}}=\left(\mathscr{P}_{\phi}\right)\left(2 t_{0}\right) 1_{\Lambda_{0}} \quad \text { a.s. }
$$

Therefore

$$
\begin{aligned}
\theta_{\mathscr{F}} f & \geq \sup _{0 \leq n<\infty}(\mathscr{P} \phi)\left(2 t_{n}\right) 1_{\Lambda_{n}} \\
& =\sum_{n=0}^{\infty}(\mathscr{P} \phi)\left(2 t_{n}\right) 1_{\Lambda_{n} \backslash \Lambda_{n+1}}=\frac{1}{2} \sum_{n=0}^{\infty}(\mathscr{P} \phi)\left(2 t_{n+1}\right) 1_{\Lambda_{n} \backslash \Lambda_{n+1}} \quad \text { a.s., }
\end{aligned}
$$

where the last equality follows from (3). Hence, for every $t \in I$,

$$
\begin{aligned}
(\theta \mathscr{F} f)^{*}(t) & \geq\left(\frac{1}{2} \sum_{n=0}^{\infty}(\mathscr{P} \phi)\left(2 t_{n+1}\right) 1_{\Lambda_{n} \backslash \Lambda_{n+1}}\right)^{*}(t) \\
& =\frac{1}{2} \sum_{n=0}^{\infty}(\mathscr{P} \phi)\left(2 t_{n+1}\right) 1_{\left[2 t_{n+1}, 2 t_{n}\right)}(t) \geq \frac{1}{2}(\mathscr{P} \phi)(t) .
\end{aligned}
$$

Since $M f=|z|$ a.s., we have $(M f)^{*}=z^{*}=\phi$. Using (i) we see that

$$
\begin{aligned}
\|\mathscr{P} \phi\|_{\widehat{X}} & \leq 2\left\|\left(\theta_{\mathscr{F}} f\right)^{*}\right\|_{\widehat{X}}=2\left\|\theta_{\mathscr{F}} f\right\|_{X} \\
& \leq 2 C\|M f\|_{Y}=2 C\left\|(M f)^{*}\right\|_{\widehat{Y}}=2 C\|\phi\|_{\widehat{Y}} .
\end{aligned}
$$

This completes the proof that (i) implies (iv), and hence the proof of Proposition 3. 
In order to prove Theorem 1, we will need one more lemma.

Lemma 4 ([5, Lemma 1]). Let $X$ be a Banach function space over $\Omega$ and let $X_{+}$denote the collection of all nonnegative random variables in $X$. Then the following are equivalent:

(i) There exists a constant $c=c(X)$ such that if $x, y \in X_{+}, x \simeq_{d} y$, and $\{x>0\} \cap\{y>0\}=\emptyset$, then $\|y\|_{X} \leq c\|x\|_{X}$.

(i) $X$ can be equivalently renormed so as to be an ri. space.

Proof of Theorem 1. As in the proof of Proposition 3, we see that (ii) implies (i) and (iii) implies (ii). We need only show that (iv) implies (iii) and (i) implies (iv).

(iv) $\Rightarrow$ (iii) Assume that (iv) holds. Then $\mathscr{P} \in B(\widehat{X})$ as mentioned at the end of Section 1. We can therefore apply Proposition 3 to derive the inequality in (iii).

(i) $\Rightarrow$ (iv) Assume that (i) holds. We first show that $X$ can be equivalently renormed so as to be an r.i. space. Assume that $x, y \in X_{+}, x \simeq_{d} y$, and $\{x>0\} \cap\{y>0\}=\emptyset$. For each $k \in Z$, let $A_{k}=\left\{2^{k} \leq x<2^{k+1}\right\}$ and $B_{k}=\left\{2^{k} \leq y<2^{k+1}\right\}$, and define random variables $x^{\prime}$ and $y^{\prime}$ by

$$
x^{\prime}=\sum_{k=-\infty}^{\infty} 2^{k} 1_{A_{k}} \quad \text { and } \quad y^{\prime}=\sum_{k=-\infty}^{\infty} 2^{k} 1_{B_{k}}
$$

Then $0 \leq x^{\prime} \leq x \leq 2 x^{\prime}, 0 \leq y^{\prime} \leq y \leq 2 y^{\prime}$, and $x^{\prime} \simeq_{d} y^{\prime}$. For each $k \in Z$, choose $A_{k, 1}$ and $A_{k, 2}$ in $\Sigma$ so that

$$
A_{k, 1} \cap A_{k, 2}=\emptyset, \quad A_{k, 1} \cup A_{k, 2}=A_{k}, \quad \text { and } \quad P\left(A_{k, 1}\right)=P\left(A_{k, 2}\right)=\frac{1}{2} P\left(A_{k}\right) .
$$

This is possible, since $\Omega$ is nonatomic. Define $\mathscr{F}=\left(\mathscr{F}_{n}\right) \in \mathrm{F}$ by

$$
\mathscr{F}_{n}= \begin{cases}\sigma\left\{A_{k} \cup B_{k} \mid k \in \mathrm{Z}\right\} & \text { if } n=0, \\ \Sigma & \text { if } n \geq 1 .\end{cases}
$$

Let $x^{\prime \prime}=\sum_{k=-\infty}^{\infty} 2^{k}\left(1_{A_{k, 1}}-1_{A_{k, 2}}\right)$ and define $f=\left(f_{n}\right) \in \mathscr{M}_{u}(\mathscr{F})$ by

$$
f_{n}=E\left[x^{\prime \prime} \mid \mathscr{F}_{n}\right]= \begin{cases}0 & \text { if } n=0, \\ x^{\prime \prime} & \text { if } n \geq 1 .\end{cases}
$$

Then, by convention, $E\left[\left|x^{\prime \prime}\right| \mid \mathscr{F}_{0}\right] \leq \theta_{\mathscr{F}} f$. On the other hand, it is straightforward to check that

$$
E\left[\left|x^{\prime \prime}\right| \mid \mathscr{F}_{0}\right]=E\left[x^{\prime} \mid \mathscr{F}_{0}\right]=\frac{x^{\prime}+y^{\prime}}{2} \geq \frac{y^{\prime}}{2} \geq \frac{y}{4} \quad \text { a.s. }
$$


Hence $y \leq 4 \theta_{\mathscr{F}} f$ a.s. Since $M f=\left|x^{\prime \prime}\right|=x^{\prime} \leq x$ a.s., it follows from (i) that $\|y\|_{X} \leq 4 C\|x\|_{X}$. Thus, by Lemma $4, X$ can be equivalently renormed so as to be an ri. space.

We can now apply Proposition 3 to see that $\mathscr{P} \in B(\widehat{X})$, or equivalently, that $\beta_{X}<1$. This completes the proof that (i) implies (iv), and hence the proof of Theorem 1 .

We conclude the paper by proving Corollary 2 .

Proof of Corollary 2. From the main theorem of [5], we know that (iii) and (iv) are equivalent.

Assume that (iii) holds, or equivalently, that (iv) holds. Then $\mathscr{Q} \in B(\widehat{X})$, and hence by [5, Proposition 3 (i)], there is a constant $C=C(X)$ such that for every $f \in \mathscr{M}_{u}(\mathscr{F})$,

$$
\sup _{0 \leq n<\infty}\left\|f_{n}\right\|_{X} \leq\|M f\|_{X} \leq C\left\|\theta_{\mathscr{F}} f\right\|_{X} .
$$

This, together with (iii), shows that the inequalities in (i) and (ii) hold. Thus (iii) implies both (i) and (ii).

Assume now that (i) or (ii) hold. Then, by Theorem 1, $X$ can be equivalently renormed so as to be an r.i. space such that $\beta_{X}<1$. Hence we can apply [5, Proposition 3 (ii)] to conclude that $\mathscr{Q} \in B(\widehat{X})$, or equivalently, that $\alpha_{X}>0$. Thus both (i) and (ii) imply (iv).

ACKNOWLEDGEMENT. We would like to thank the referee for a careful reading of the manuscript and for helpful suggestions.

\section{REFERENCES}

1. Bennett, C., and Sharpley, R., Interpolation of Operators, Pure Appl. Math. 129, Academic Press, Boston 1988.

2. Chong, K. M., and Rice, N. M., Equimeasurable Rearrangements of Functions, Queen's Papers in Pure and Appl. Math. 28, Queen's Univ., Kingston, Ont. 1971.

3. Kikuchi, M., Characterization of Banach function spaces that preserve the Burkholder squarefunction inequality, Illinois J. Math. 47 (2003), 867-882.

4. Kikuchi, M., New martingale inequalities in rearrangement-invariant function spaces, Proc. Edinb. Math. Soc. (2) 47 (2004), 633-657.

5. Kikuchi, M., On some mean oscillation inequalities for martingales, Publ. Mat. 50 (2006), 167-189.

6. Lépingle, D., Quelques inégalités concernant les martingales, Studia Math. 59 (1976), 63-83.

7. Long, R. L., Rearrangement techniques in martingale setting, Illinois J. Math. 35 (1991), 506-521.

8. Long, R. L., Martingale Spaces and Inequalities, Peking Univ. Press, Beijing 1993.

9. Luxemburg, W. A. J., Rearrangement-invariant Banach function spaces, pp. 83-144 in: Proceedings of the Symposium in Analysis, Queen's Papers in Pure and Appl. Math. 10, Queen's Univ., Kingston, Ont. 1967. 
10. Shimogaki, T., Hardy-Littlewood majorants in function spaces, J. Math. Soc. Japan 17 (1965), 365-373.

\author{
DEPARTMENT OF MATHEMATICS \\ UNIVERSITY OF TOYAMA \\ 3190 GOFUKU \\ 930-8555 TOYAMA \\ JAPAN \\ E-mail: kikuchi@sci.u-toyama.ac.jp
}

